\title{
ATUAÇÃO FISIOTERAPÊUTICA CORRELACIONADA A MONITORIZAÇÃO EM PACIENTES COM HIPERTENSÃO INTRACRANIANA NA UNIDADE DE TERAPIA INTENSIVA: REVISÃO DE LITERATURA
}

\section{ARTIGO DE REVISÃO}

\author{
ALBUQUERQUE, Dayanne Cunha ${ }^{1}$
}

SANTOS, Andrea Kerckhoff ${ }^{2}$

ALBUQUERQUE, Dayanne Cunha. SANTOS, Andrea Kerckhoff. Atuação fisioterapêutica correlacionada a monitorização em pacientes com Hipertensão Intracraniana na Unidade de Terapia Intensiva: Revisão de literatura. Revista Científica Multidisciplinar Núcleo do Conhecimento. Ano 04, Ed. 10, Vol. 06, pp. 3348. Outubro de 2019. ISSN: 2448-0959, Link de acesso: https://www.nucleodoconhecimento.com.br/saude/atuacao-fisioterapeutica

\section{RESUMO}

A incidência de hipertensão intracraniana é frequente em unidades de terapia intensiva. Devido à grave lesão cerebral, o tratamento fisioterapêutico deve ser realizado com cautela, seguindo recomendações específicas. Objetivo: Descrever os principais estudos sobre a atuação da fisioterapia intensiva correlacionando a monitorização de pacientes acometidos por hipertensão intracraniana. Metodologia: Foi realizada revisão de literatura por meio das bases de dados Pubmed, Scielo e Google Scholar, utilizando as conformações "Hipertensão Intracraniana", "Fisioterapia Intensiva", Monitorização da Pressão Intracraniana". Foram selecionados materiais que abordaram o tema pesquisado entre 2005 e 2017,

\footnotetext{
${ }_{1}^{1}$ Pós-graduada em Fisioterapia em Terapia Intensiva. Graduada em Fisioterapia.

2 Professora da Faculdade Inspirar Vitória, Mestre em Ciência da Motricidade Humana (Vitória/ Espírito Santo/ Brasil).
} 
nos idiomas português e inglês. Resultados: A fisioterapia intensiva aliada à monitorização permite que diversas intervenções respiratórias e motoras sejam realizadas com segurança em pacientes acometidos por hipertensão intracraniana. Conclusão: Através da coleta de dados da monitorização da pressão intracraniana, pressão intra-abdominal, pressão intratorácica, pressão de perfusão cerebral, pressão venosa cerebral, pressão arterial média, pressão parcial de oxigênio e pressão parcial de dióxido de carbono, é possível realizar manobras de higiene brônquica, expansão pulmonar, posicionamento terapêutico, exercícios metabólicos leves e aspiração endotraqueal em pacientes com hipertensão intracraniana igual ou menor a $30 \mathrm{mmHg}$. Sendo a aspiração endotraqueal um procedimento fonte de opiniões controversas, sugerindo maior cuidado em sua execução.

Palavras-chaves: Hipertensão Intracraniana, fisioterapia intensiva, fisioterapia respiratória, monitorização da pressão intracraniana, unidade de terapia intensiva.

\section{INTRODUÇÃO}

Sabe-se que a estrutura cerebral é limitada pelos ossos do crânio, dividindo espaço com o líquido cérebro raquidiano e os vasos presentes no encéfalo. Essa restrição é implacável, caso ocorram distúrbios graves, como tumores, traumas cranioencefálicos ou edema, o organismo não consegue dar conta de redistribuir líquido e sangue venoso de forma eficaz, levando ao aumento da pressão intracraniana. (FILHO, 2011).

Ao tratar pacientes adultos, considera-se hipertensão intracraniana uma pressão local mantida a $20 \mathrm{mmHg}$ (milímetros de mercúrio) por mais de vinte minutos. Hemodinamicamente, são ditos parâmetros ideais uma pressão intracraniana de 10 $\mathrm{mmHg}$, pressão de perfusão cerebral maior que $70 \mathrm{mmHg}$ e uma pressão arterial média por volta de 70 a $110 \mathrm{mmHg}$.(TOLEDO et al, 2008).

A elevação da pressão intracraniana é dada pelo aumento da pressão venosa do cérebro, queda da drenagem venosa local e do plexo lombar, comprimindo mecanicamente a veia cava inferior. (FALCÃO; OLIVEIRA, 2011). 
Para diagnosticar a hipertensão intracraniana, somente os sinais clínicos de cefaléia, edema papilar ótico, escala de coma Glasgow igual ou menor a oito, vômitos e resposta de Cushing não são suficientes, pois eles ocorrem quando o quadro se agrava. Dessa forma, são necessários instrumentos de avaliação, para que a hipertensão intracraniana seja diagnosticada de forma rápida e precisa, e tão logo seja iniciado o tratamento adequado. Através da tomografia computadorizada, podem ser observadas lesões expansivas, com desvio das estruturas, presença de transudato ventricular, impedindo visualização dos ventrículos laterais bem como o terceiro ventrículo. Pode ser realizada também a ultrassonografia do nervo óptico, que se apresenta com a bainha de mielina distendida em pacientes com hipertensão intracraniana aguda. (PAPALINI, 2018).

O método mais eficaz de diagnosticar a hipertensão intracraniana é através da inserção de um cateter no ventrículo, no espaço subaracnóideo ou no parênquima. De estrutura semi-rígida, ele possui um transdutor em sua extremidade, conectado ao monitor por uma fibra ótica, sendo possível registrar continuamente os valores da pressão intracraniana do paciente. Por ser altamente invasivo, necessita de tempo para inserção, cuidados com a manutenção, e pode causar adversidades como infecções, disfunções neurológicas e sangramentos. (PAPALINI, 2018).

Sabe-se que tanto a elevação da pressão intra abdominal quanto a elevação da pressão intratorácica podem culminar no aumento da pressão intracraniana. Sendo necessária atenção a essas variáveis. (FALCÃO; OLIVEIRA, 2011).

É conhecido que o excesso de oxigênio é deletério, pois ele causa a diminuição do fluxo sanguíneo de forma reflexa, aumentando a pressão venosa central e conseqüentemente elevando a pressão intracraniana. (FILHO, 2011).

Por outro lado, não se deve acumular dióxido de carbono, pois em quantidade superior à recomendada, pode levar ao relaxamento dos vasos cerebrais, aumentando o extravasamento de líquido, pressão venosa central e novamente, subindo a pressão intracraniana. (FILHO, 2011). 
O paciente que apresenta hipertensão intracraniana tem baixo nível de consciência, sendo incapaz de proteger e ventilar corretamente as vias aéreas. Por isso, a ventilação mecânica invasiva é indispensável. O ventilador fornece dois tipos de pressões: pressão inspiratória positiva e pressão expiratória final positiva. (ABREU; ALMEIDA, 2009; TOLEDO et al, 2008).

A pressão inspiratória permite a entrada do ar nos pulmões, simulando a inspiração, de forma passiva. Permite a oxigenação do corpo, prevenindo o sofrimento de tecidos e órgãos. (CARVALHO; FERREIRA; COSTA, 2015).

A pressão expiratória final positiva (PEEP) tem efeito de elevar a capacidade residual funcional, deixando uma parte do ar nos pulmões ao final da expiração, excelente para a manutenção da expansão pulmonar. Ela também reduz a necessidade de oferta de oxigênio, o que diminui os riscos de lesão pulmonar. (DIRETRIZES BRASILEIRAS DE VENTILAÇÃO MECÂNICA, 2013).

Com o benefício do uso da ventilação mecânica invasiva, se fazem necessárias a higienização e aspiração do tubo endotraqueal, para evitar o acúmulo de secreções, que podem causar desde a obstrução do circuito, infecções que em casos graves, podem levar ao óbito do paciente. Em casos onde se encontra em grande quantidade, não apresenta fluidez ou está em áreas ditas iniciais dos pulmões, o fisioterapeuta precisará utilizar manobras de higiene brônquica, para que consiga fazer a remoção com sucesso. (CARVALHO; FERREIRA; COSTA, 2015).

Dentre as diversas terapias que o paciente diagnosticado com hipertensão intracraniana irá se beneficiar em uma unidade de terapia intensiva, a fisioterapia tem o seu papel definido, sendo importante para a preservação das vias aéreas e prevenção de injúrias secundárias. (TOLEDO et al, 2008).

O paciente com hipertensão intracraniana encontra-se grave, deitado em um leito de unidade de terapia intensiva, sedado. Cuidados com o posicionamento do mesmo também fazem parte da rotina do fisioterapeuta, prevenindo úlceras de pressão, deformações, protegendo funções. (SOUZA, ZEDAN, 2013). 
Muito comum em unidades de terapia intensiva neurológica, o manejo da hipertensão intracraniana é um desafio para o fisioterapeuta intensivo, que deve monitorar os efeitos das suas intervenções terapêuticas sobre a pressão intracraniana do seu paciente. (SABACK et al, 2007).

Não foram encontrados estudos que abordassem a atuação fisioterapêutica em pacientes com hipertensão intracraniana acima de $30 \mathrm{mmHg}$. Porém, orienta-se que a fisioterapia intensiva tanto respiratória quanto motora não seja realizada em nestes casos, devido ao risco de morte encefálica. (SABACK et al, 2007, PADOVANI, 2015, DOREA et al, 2014).

Existem poucos estudos que tratam da atuação da fisioterapia intensiva correlacionada a monitorização da hipertensão intracraniana. Por muito tempo acreditava-se que não havia muito a fazer nesses casos, devido à gravidade em que se apresentam. Atualmente, sabe-se que o fisioterapeuta tem muito a colaborar com a equipe multidisciplinar para promover o tratamento e reabilitação desses pacientes, desde que haja extrema cautela. $O$ presente artigo tem como objetivo investigar os principais estudos que informem sobre a atuação do fisioterapeuta usando os dados da monitorização de pacientes com hipertensão intracraniana, buscando descrever a forma segura de realizar as intervenções necessárias.

\section{METODOLOGIA}

Para a elaboração deste trabalho adotou-se a técnica de um estudo descritivo, utilizando dados encontrados na literatura já existente. Foram realizadas buscas por publicações e documentos sobre o assunto pesquisado, nos diversos veículos disponíveis impressos ou digitais: jornais, artigos científicos, dissertações, livros, monografias, Pubmed, Google Scholar, Scielo e revistas. Utilizando as conformações "Hipertensão Intracraniana", "Fisioterapia Intensiva", Monitorização da Pressão Intracraniana" "Intracranial Hypertension", "Intensive Physiotherapy", "Monitoring of Intracranial Pressure". 
As buscas ocorreram de janeiro a julho de 2018 e foram incluídos dez artigos de revisões sistemáticas, estudos prospectivos e estudos de caso, que abordaram a fisioterapia intensiva correlacionada a monitorização de pacientes com hipertensão intracraniana no período de 2005 a 2017, nos idiomas português e inglês. Foram excluídos artigos publicados com data inferior a 2005.

\section{RESULTADOS}

Após o estudo dos artigos selecionados, os dados foram organizados em formato de tabela, que contém nome de autores, ano de publicação em ordem decrescente, descrição da pesquisa e resultados obtidos. Todos esses dados estão expostos na Tabela 1.

Tabela 1- Descrição dos estudos revisados.

\begin{tabular}{|c|c|c|}
\hline Autores/Ano & Estudo realizado & Resultados Obtidos \\
\hline Silva, 2017. & $\begin{array}{l}\text { Estudo observacional analítico } \\
\text { transversal, trinta e cinco } \\
\text { pacientes com traumatismo } \\
\text { cranioencefálico (três com } \\
\text { cateter de monitorização de } \\
\text { pressão intracraniana). Foram } \\
\text { colhidos dados de pressão } \\
\text { intracraniana, pressão arterial } \\
\text { média e pressão de perfusão } \\
\text { cerebral submetidos a } \\
\text { manobras de higiene brônquica } \\
\text { e expansão pulmonar (manobra } \\
\text { de pressão negativa, } \\
\text { compressão manual e uso do } \\
\text { ambú para hiperinsuflação } \\
\text { manual, aspiracão endotraqueal }\end{array}$ & $\begin{array}{l}\text { Durante as manobras de } \\
\text { fisioterapia respiratória, a } \\
\text { pressão intracraniana inicial } \\
\text { era de } 11,33 \mathrm{mmHg} \text {, durante } \\
\text { atendimento subiu para } 23 \\
\text { mmHg, caiu para } 11 \mathrm{mmHg} \\
\text { imediatamente após o término } \\
\text { e após cinco minutos manteve- } \\
\text { se em } 14,66 \mathrm{mmHg} \text {. A pressão } \\
\text { de perfusão cerebral inicial de } \\
57,66 \quad \text { mmHg, durante } \\
\text { atendimento caiu para } 45,66 \\
\text { mmHg, imediatamente após o } \\
\text { término subiu para } 63,66 \\
\text { mmHg, e cinco minutos após o } \\
\text { término manteve-se em } 54,66\end{array}$ \\
\hline
\end{tabular}




\begin{tabular}{|c|c|c|}
\hline & 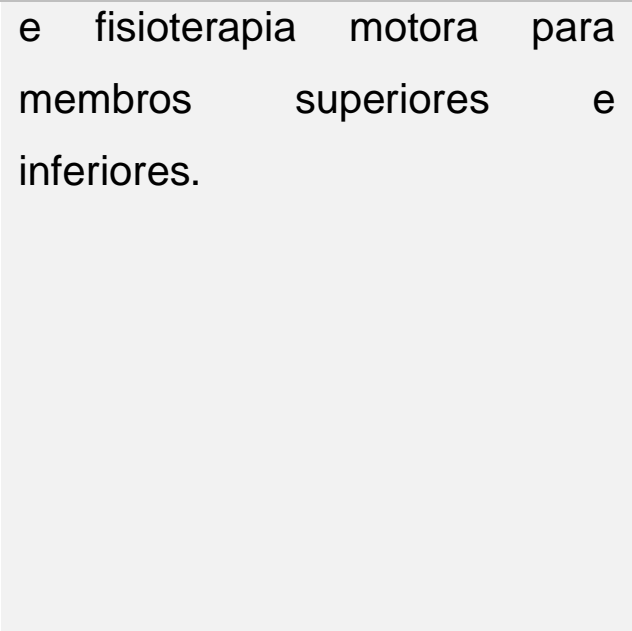 & $\begin{array}{l}\text { mmHg. A pressão arterial } \\
\text { média inicial era de } 82 \mathrm{mmHg} \text {, } \\
\text { durante o atendimento subiu } \\
\text { para } 86 \mathrm{mmHg} \text {, retornando ao } \\
\text { valor basal imediatamente } \\
\text { após o término do } \\
\text { atendimento, e caindo para } 78 \\
\text { mmHg cinco minutos após o } \\
\text { término. }\end{array}$ \\
\hline $\begin{array}{l}\text { PADOVANI, } \\
2015 .\end{array}$ & $\begin{array}{l}\text { Revisão de literatura } \\
\text { sistemática, que descreveu } \\
\text { cinco artigos sobre fisioterapia } \\
\text { respiratória no traumatismo } \\
\text { cranioencefálico. }\end{array}$ & $\begin{array}{l}\text { Manobras como vibro } \\
\text { compressão manual } \\
\text { aceleração do fluxo expiratório } \\
\text { não alteram significativamente } \\
\text { a pressão intracraniana em } \\
\text { paciente com hipertensão } \\
\text { intracraniana de até } 30 \text { mmHg } \\
\text { (milímetros de mercúrio). Já a } \\
\text { aspiração endotraqueal } \\
\text { promove o aumento transitório } \\
\text { da pressão intracraniana, com } \\
\text { retorno basal após dez } \\
\text { minutos. }\end{array}$ \\
\hline $\begin{array}{l}\text { ALMEIDA et al, } \\
2014 .\end{array}$ & $\begin{array}{l}\text { Pesquisa qualitativa, } \\
\text { exploratória e descritiva, reuniu } \\
\text { artigos que tratam dos efeitos da } \\
\text { fisioterapia respiratória e } \\
\text { aspiração endotraqueal em } \\
\text { pacientes com hipertensão } \\
\text { intracraniana. }\end{array}$ & $\begin{array}{l}\text { Fisioterapia respiratória e } \\
\begin{array}{l}\text { aspiração endotraqueal } \\
\text { causam pequeno e passageiro } \\
\text { aumento da } \quad \text { pressão } \\
\text { intracraniana, sem alterar } \\
\text { significativamente a pressão } \\
\text { de perfusão cerebral. }\end{array}\end{array}$ \\
\hline
\end{tabular}




\begin{tabular}{|c|c|c|}
\hline $\begin{array}{l}\text { DOREA; } \\
\text { COSTA; RUIZ, } \\
2014 .\end{array}$ & $\begin{array}{l}\text { Revisão de literatura descreveu } \\
\text { dezenove artigos sobre o uso de } \\
\text { pressão expiratória positiva final } \\
\text { em pacientes com traumatismo } \\
\text { cranioencefálico. }\end{array}$ & $\begin{array}{l}\text { Valores de } 0 \text { a } 15 \text { cmH20 } \\
\text { (centímetros de água) foram } \\
\text { considerados seguros, por não } \\
\text { alterarem significativamente a } \\
\text { pressão arterial média, } \\
\text { pressão intracraniana } \\
\text { Pressão de perfusão cerebral. }\end{array}$ \\
\hline $\begin{array}{l}\text { FERREIRA; } \\
\text { VALENTINI; } \\
\text { VANDERLEI, } \\
2013 .\end{array}$ & $\begin{array}{l}\text { Revisão sistemática sobre } \\
\text { fisioterapia respiratória na } \\
\text { pressão intracraniana de } \\
\text { pacientes graves. }\end{array}$ & $\begin{array}{l}\text { Quase unanimidade de } \\
\text { estudos mostrando aumento } \\
\text { transitório da } \\
\text { intracraniana } \\
\text { manobras de }\end{array} \begin{array}{r}\text { durante } \\
\text { brônquica } \\
\text { endotraqueal, seme }\end{array}$ \\
\hline $\begin{array}{l}\text { CERQUEIRA- } \\
\text { NETO et al, } \\
2013 .\end{array}$ & $\begin{array}{l}\text { Ensaio clínico do tipo } \\
\text { prospectivo, vinte pacientes } \\
\text { submetidos a manobras de } \\
\text { fisioterapia respiratória (vibro } \\
\text { compressão, aumento de fluxo } \\
\text { expiratório) e aspiração } \\
\text { endotraqueal foram monitorados } \\
\text { durante as primeiras quarenta e } \\
\text { oito horas de internação. }\end{array}$ & $\begin{array}{l}\text { Houve manutenção da } \\
\text { hemodinâmica basal durante } \\
\text { as manobras. Já durante a } \\
\text { aspiração endotraqueal } \\
\text { observou-se instabilidade } \\
\text { hemodinâmica com elevação } \\
\text { da pressão intracraniana e } \\
\text { pressão arterial média durante } \\
\text { até dez minutos do final da } \\
\text { técnica. }\end{array}$ \\
\hline $\begin{array}{l}\text { SOUZA; } \\
\text { ZEDAN, } 2013 .\end{array}$ & $\begin{array}{l}\text { Estudo de caso de paciente } \\
\text { sexo masculino, quarenta e três } \\
\text { anos, com traumatismo } \\
\text { cranioencefálico grave, nível } \\
\text { três na escala de Glasgow, em } \\
\text { ventilação mecânica invasiva, }\end{array}$ & $\begin{array}{l}\text { Melhora do padrão } \\
\text { respiratório, melhora da } \\
\text { propriocepção abdominal, do } \\
\text { controle de cabeça quando } \\
\text { sentado e estímulos visuais, }\end{array}$ \\
\hline
\end{tabular}




\begin{tabular}{|c|c|c|}
\hline & $\begin{array}{l}\text { foi submetido a vinte sessões de } \\
\text { fisioterapia respiratória e } \\
\text { motora. }\end{array}$ & $\begin{array}{l}\text { sem interferir na pressão } \\
\text { intracraniana. }\end{array}$ \\
\hline $\begin{array}{l}\text { TOLEDO et al, } \\
2008 .\end{array}$ & $\begin{array}{l}\text { Ensaio prospectivo clínico } \\
\text { intervencional, onze pacientes } \\
\text { com lesão cerebral agudam que } \\
\text { foram submetidos a manobras } \\
\text { de fisioterapia respiratória } \\
\text { tiveram os dados da pressão } \\
\text { intracraniana e pressão arterial } \\
\text { média anotados antes, durante } \\
\text { e após os procedimentos. }\end{array}$ & $\begin{array}{l}\text { Após o terceiro dia de manobra } \\
\text { de vibração manual bilateral } \\
\text { houve queda significativa da } \\
\text { pressão intracraniana. Já a } \\
\text { compressão torácica causou } \\
\text { aumento significativo, porém } \\
\text { transitório da pressão } \\
\text { intracraniana, aumento } \\
\text { compensatório da pressão de } \\
\text { perfusão cerebral, que impediu } \\
\text { a elevação da pressão } \\
\text { intracraniana. }\end{array}$ \\
\hline $\begin{array}{l}\text { SABACK; } \\
\text { ALMEIDA; } \\
\text { ANDRADE, } \\
2007 .\end{array}$ & $\begin{array}{l}\text { Estudo descritivo entrevistou } \\
\text { setenta fisioterapeutas } \\
\text { intensivos de Salvador, Bahia, } \\
\text { questionando como eles } \\
\text { ventilam pacientes com trauma } \\
\text { cranioencefálico e síndrome do } \\
\text { desconforto respiratório agudo. }\end{array}$ & $\begin{array}{l}91,4 \% \text { utilizam o modo pressão } \\
\text { controlada para ventilar. } 68,6 \% \\
\text { mantém a pressão parcial de } \\
\text { dióxido de carbono entre } 30 \text { e } \\
35 \mathrm{mmHg} \text {. } 44,3 \% \text { usam a } \\
\text { pressão positiva expiratória } \\
\text { final ideal como a que forneça } \\
\text { a melhor saturação de oxigênio } \\
\text { com a menor fração inspirada } \\
\text { de oxigênio possível. } 64,3 \% \\
\text { não possuem monitorização de } \\
\text { pressão intracraniana na } \\
\text { unidade de terapia intensiva } \\
\text { em que trabalham. } 62,4 \% \text { não } \\
\text { possuem monitorização de } \\
\text { capnografia. }\end{array}$ \\
\hline
\end{tabular}




\begin{tabular}{|c|c|c|}
\hline & 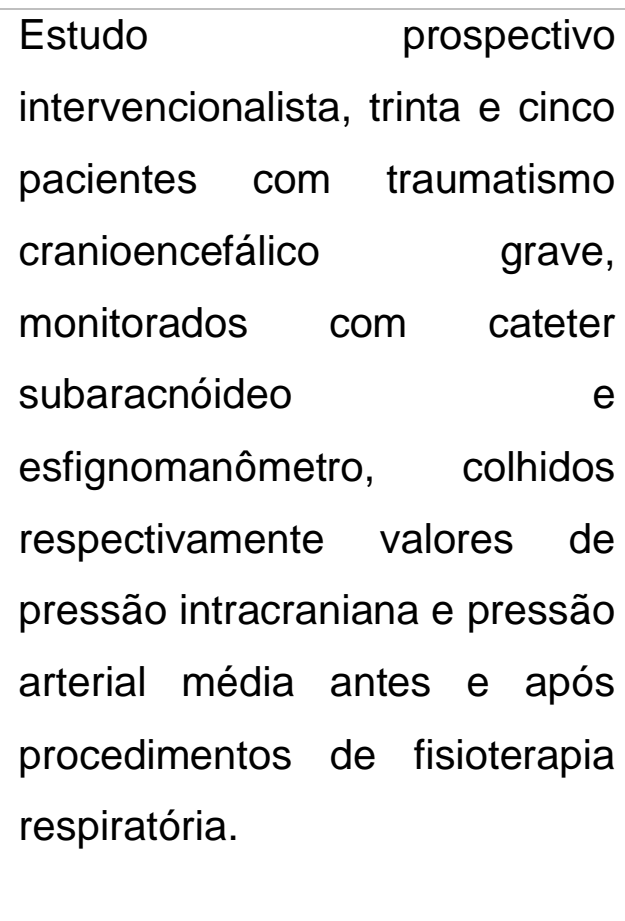 & $\begin{array}{l}\text { Houve considerável aumento } \\
\text { da pressão intracraniana após } \\
\text { aspiração endotraqueal, } \\
\text { compressão manual, vibração } \\
\text { diafragmática e drenagem } \\
\text { postural associada à } \\
\text { tapotagem. O retorno aos } \\
\text { valores basais ocorreu após } \\
\text { trinta minutos. A pressão } \\
\text { arterial média e pressão de } \\
\text { perfusão cerebral não } \\
\text { sofreram alterações } \\
\text { significativas. }\end{array}$ \\
\hline
\end{tabular}

\section{DISCUSSÃO}

Para Saback et al (2007), ao tratar um paciente com hipertensão intracraniana admitido em unidade de terapia intensiva, não se deve pensar somente na fisioterapia respiratória tecnicamente, faz-se necessário saber as interferências que as técnicas aplicadas causam na hemodinâmica do paciente, que podem repercutir negativamente no compartimento cerebral do mesmo.

Não foram encontrados estudos que elegessem o modo controlado ideal para ventilar pacientes com hipertensão intracraniana. Porém, Saback et al (2007) e Lessa (2011) corroboram que o mais utilizado é o controlado à pressão, por permitir o estabelecimento de baixas pressões. Recomenda-se uma pressão de pico máxima de 25 a $50 \mathrm{cmH} 2 \mathrm{O}$ e uma pressão de platô entre 15 e $35 \mathrm{~cm} \mathrm{H} 2 \mathrm{O}$, mantendo uma ventilação protetora.

Porém, foi observado ainda por Lessa (2011), que pacientes com hipertensão intracraniana internados em unidade de terapia intensiva, ventilados com modo controlado à pressão, estavam fazendo volumes correntes acima dos padrões normais, que podem elevar a pressão intratorácica, diminuir o retorno venoso 
encefálico e elevar a pressão intracraniana. Não foram encontrados estudos com os demais modos ventilatórios.

Assim como a pressão inspiratória, a pressão expiratória ao final da expiração deve ser a mais baixa possível que consiga manter uma adequada expansão pulmonar. Backaus et al (2017), Boone et al (2017) e Dorea et al (2014) corroboram sobre o uso seguro da PEEP. Backaus et al (2017) verificou através de ecografia duplex transcraniana, que valores de PEEP de $05 \mathrm{~cm} \mathrm{H2O,} 07 \mathrm{~cm} \mathrm{H} 20$ e $10 \mathrm{~cm} \mathrm{H} 20$ não causaram alterações significativas no fluxo sanguíneo cerebral de sete pacientes. Somente em casos especiais de herniação cerebral e hipertensão intracraniana refratária com hiperemia são avaliados valores maiores, para uma hiperventilação.

Em seu estudo, Boone et al (2017) reforça que através de dados retrospectivos coletados de 341 pacientes com lesão cerebral aguda, foi observado que para cada centímetro de água de aumento na PEEP, houve uma aumento de 0,31 centímetro de água na pressão intracraniana e uma queda de 0,85 milímetros de mercúrio na pressão de perfusão cerebral, sugerindo que a PEEP pode ser aplicada com segurança.

Para Dorea (2014), valores de PEEP de 0 a $15 \mathrm{~cm} \mathrm{H} 2 \mathrm{O}$ são seguros em pacientes com até $30 \mathrm{mmHg}$ de hipertensão intracraniana, pois não causam alterações significativas da pressão arterial média, pressão de perfusão cerebral e pressão intracraniana.

Segundo Filho (2011), tanto a pressão inspiratória quanto a PEEP em números acima do recomendado podem levar a hipertensão intratorácica. Essa por sua vez pode aumentar a pressão venosa cerebral, diminuir o débito cardíaco, levar à queda da pressão arterial média e da pressão de perfusão cerebral, tendo como resultado o aumento da pressão intracraniana.

Os autores Filho (2011) e Saback et al (2007) concordaram que utilizando o benefício da ventilação mecânica invasiva, almeja-se a manutenção de uma saturação de oxigênio entre 92 e 94\%. O excesso de oxigênio é deletério por causar diminuição 
reflexa do fluxo sanguíneo, aumentando a pressão venosa cerebral, que culminará no aumento da pressão intracraniana. Devem ser adotadas então, baixas frações inspiradas de oxigênio, que sejam suficientes para manter os valores citados de saturação de oxigênio.

É necessária também atenção ao dióxido de carbono. O ideal é manter a pressão de dióxido de carbono entre 35 e $40 \%$, pois o excesso dele causa o relaxamento dos vasos cerebrais, extravasamento de líquido para o interstício, sobrecarga venosa e o final é o mesmo: aumento da pressão intracraniana. Para garantir o controle do dióxido de carbono deve-se certificar que a exalação do ar está sendo realizada de forma plena pelo circuito. Segundo Filho (2011) retenções de dióxido de carbono podem ser causadas por obstruções da saída do ar, ou até mesmo número de respirações programadas insuficientes. Para monitorização, existe o capnógrafo e a gasometria arterial, ambos capazes de fornecer os valores da pressão parcial de dióxido de carbono.

Para Silva (2017), Padovani (2015) e Cerqueira-neto (2013) manobras de pressão negativa, compressão manual, hiperinsuflação manual, aceleração do fluxo expiratório, manobras de expansão pulmonar e vibro compressão não alteram significativamente a pressão intracraniana, em pacientes com até $30 \mathrm{mmHg}$.

Já Toledo et al (2008), verificou queda significativa na pressão intracraniana no terceiro dia de manobra de vibração manual bilateral. E aumento significativo, porém passageiro da pressão intracraniana e pressão de perfusão cerebral após realizar compressão torácica.

Ao realizar as técnicas de compressão manual, vibração diafragmática, drenagem postura e tapotagem, Thiesen et al (2005) identificou aumento da pressão intracraniana durante trinta minutos, sem interferir na pressão arterial média e pressão de perfusão cerebral.

Foram encontradas divergências sobre os impactos da aspiração endotraqueal em pacientes com hipertensão intracraniana. Silva (2017), não observou alterações 
significativas na pressão intracraniana durante e após o procedimento, relatando ainda, uma queda de quatro milímetros de mercúrio na pressão arterial média cinco minutos após o fim do atendimento.

Já Cerqueira-neto (2013), observou instabilidade hemodinâmica, aumento da pressão intracraniana e pressão arterial média por até dez minutos do término do procedimento.

Em seu estudo, Padovani (2015) fala em aumento transitório da pressão intracraniana, com duração de dez minutos após o encerramento da técnica. Para Thiesen et al (2005) houve aumento considerável da pressão intracraniana, que se manteve por trinta minutos após o fim da técnica. Ambos recomendam que a técnica seja realizada de forma rápida, somente quando haja real necessidade. Além disso é necessário verificar se o nível de sedação e analgesia está suficiente, para não causar reflexos de tosse e proteção, que podem aumentar a pressão intracraniana.

Os autores Almeida (2014) e Ferreira et al (2013) corroboram sobre a segurança do procedimento de aspiração endotraqueal, citando um aumento pequeno e transitório da pressão intracraniana, sem repercussões na pressão de perfusão cerebral.

Segundo Saback et al (2007), o fisioterapeuta intensivo deve cuidar também do posicionamento do paciente com hipertensão intracraniana no leito da unidade de terapia intensiva. A cabeceira da cama deve ser mantida com elevação de 30, com a cabeça centralizada e os membros inferiores e superiores neutros, descansados. Essa postura impede que o corpo e a gravidade exerçam demasiada pressão sobre o abdômen e tórax, evitando uma hipertensão abdominal e torácica.

Em seu estudo, Filho (2011) informa sobre a necessidade de minimizar as perdas musculares e circulatórias que o paciente acamado sofre. A fisioterapia motora desde que acompanhada da monitorização hemodinâmica geral do paciente, trará ótimos resultados. Exercícios passivos metabólicos de membros superiores e inferiores de pouca amplitude podem ser realizados. Lembrando que é preciso ter cautela na execução, para não causar aumento das pressões abdominal, intratorácica já que têm 
relação direta com a pressão intracraniana. Souza e Zedan (2013) observaram melhora da propriocepção e controle de cabeça na posição sentado, após programa de fisioterapia motora. Assim como Silva (2017), não ocorreram alterações significativas da pressão intracraniana durante $o$ atendimento.

\section{CONCLUSÃO}

A colaboração do fisioterapeuta intensivo é parte indispensável do tratamento e reabilitação do paciente acometido por hipertensão intracraniana. Através da coleta de dados da monitorização da pressão intracraniana, pressão intra-abdominal, pressão intratorácica, pressão de perfusão cerebral, pressão venosa cerebral, pressão arterial média, pressão parcial de oxigênio e dióxido de carbono, é obtida segurança para realizar suas intervenções terapêuticas.

Foi evidenciado que é recomendado realizar manobras de higiene brônquica, expansão pulmonar, posicionamento, exercícios metabólicos leves e aspiração endotraqueal quando a hipertensão intracraniana é menor ou igual a $30 \mathrm{mmHg}$. Os exercícios metabólicos de membros superiores e inferiores, bem como o posicionamento terapêutico no leito mostraram-se as técnicas mais seguras, que não interferiram negativamente na pressão intracraniana. Durante as manobras de fisioterapia respiratória estudadas, é percebido o aumento da pressão intracraniana de pequena e média magnitude, porém os valores são normalizados rapidamente após o fim da intervenção. Já a aspiração endotraqueal mostrou-se a técnica mais arriscada e controversa, que requer maior atenção, rapidez e destreza de quem a executa.

Devido à escassez de materiais disponíveis, sugere-se que sejam elaborados mais estudos sobre o tema, para sedimentar o conhecimento científico sobre a atuação do fisioterapeuta correlacionada à monitorização da hipertensão intracraniana. 


\section{REFERÊNCIAS}

ABREU, M. O, ALMEIDA, L. Manuseio de ventilação mecânica no trauma cranioencefálico: hiperventilação e pressão positiva expiratória final. Revista Brasileira de Terapia Intensiva, Salvador, 21 (1) p 72-79, 2009.

ALMEIDA, J. A. S. P. et al. As repercussões da aspiração traqueal e da fisioterapia respiratória na hipertensão intracraniana: um estudo de revisão. Revista Connectionline, Mato Grosso, 11 p 73-84, 2014.

BACKAUS, R. et al. Influence of positive end-expiratory pressure ventilation on cerebral perfusion and cardiac hemodynamics. Journal of Depression and Anxiety, Alemanha, 6 (3), 2017.

BARBAS et al. Diretrizes Brasileiras de Ventilação Mecânica. Associação de Medicina Intensiva Brasileira, 2013.

BOONE, M. D. et al. The effect of positive end-expiratory pressure on intracranial pressure and cerebral hemodynamics. Neurocritical Care, Estados Unidos, 26 (2) o 174-181, 2017.

CARVALHO, Roberto. R. de. FERREIRA, J. C. COSTA, E. L. V. Ventilação mecânica princípios e aplicação. Ed. 2. Atheneu.

DOREA, T. COSTA, L. RUIZ, M. P. Utilização de pressão positiva expiratória final em pacientes com traumatismo cranioencefálico. Disponível em: https://www.repositorio.bahiana.edu.br:8443/jspui/bitstream/bahiana/350/1/TCC\%20 POS\%20BAHIANA.pdf 22/01/2018.

FALCÃO, A. L. E, OLIVEIRA, D. G. Hipertensão intra-abdominal associada à lesão pulmonar aguda: efeitos sobre a pressão intracraniana. Revista Brasileira de Terapia Intensiva, São Paulo, 23 (2) p 117-119, 2011. 
FERREIRA, Lucas. L. VALENTI, V. E. VANDERLEI, L. C. M. Fisioterapia respiratória na pressão intracraniana de pacientes graves internados em unidade de terapia intensiva: revisão sistemática. Revista Brasileira de Terapia Intensiva, São Paulo, 25 (4) p 327-333, 2013.

FILHO, G. T. H. Tratamento da hipertensão intracraniana. Revista Portuguesa de Terapia Intensiva, Recife, 18 (3) p 39-46, 2011.

LESSA, R. L. Avaliação do volume corrente em pacientes neurocríticos ventilados no modo pressórico em uma unidade de terapia intensiva. Sociedade Brasileira de Terapia Intensiva. Disponível em: http://aneste.org/avaliaco-do-volume-corrente-empacientes-neurocrticos-ventilad.html Acesso em 14/02/2018.

OLIVEIRA, E. et al. Traumatismo crânio-encefálico: abordagem integrada. Acta Médica Portuguesa, São Paulo, 25 (3) p 179-192, 2012.

PADOVANI, C. Fisioterapia Respiratória no Traumatismo Cranioencefálico: Revisão de Literatura. Revista Inspirar Movimento \& Saúde, São Paulo, 35 (7) p 11-14, 2015.

PAPALINI, E. P. Nervo óptico: medida do diâmetro de sua bainha para detectar hipertensão intracraniana. Revista Brasileira de Oftalmologia, Minas Gerais, 77 (2) p 68-71, 2018.

SABACK, L. M. P et al. Trauma cranioencefálico e síndrome do desconforto respiratório agudo: Como ventilar? Avaliação da prática clínica. Revista Brasileira de Terapia Intensiva, Salvador, 19 (1) p 44-52, 2007.

SILVA, N. F. Efeitos da fisioterapia respiratória e da mobilização precoce em pacientes com traumatismo crânio encefálico. Universidade Federal de Uberlândia. Disponível em:

https://repositorio.ufu.br/bitstream/123456789/20754/5/EfeitosFisioterapiaRespirat\% C3\%B3ria.pdf 15/01/2018. 
SOUZA, R. J, ZEDAN, R. Assistência Fisioterapêutica a pacientes com traumatismo cranioencefálico (TCE) em unidade de terapia intensiva (UTI): relato de caso. Revista Hórus, São Paulo, 8 (2) p 21-28, 2013.

THIESEN R. A, et al. Influência da fisioterapia respiratória na pressão intracraniana em pacientes com traumatismo craniano grave. Arq Neuropsiquiatria, São Paulo, 3 (1) p 110-113, 2005.

TOLEDO, Cássia et al. Efeitos da fisioterapia respiratória na pressão intracraniana e pressão de perfusão cerebral no traumatismo cranioencefálico grave. Revista Brasileira de Terapia Intensiva, São Paulo, 20 (4) p 339-343, 2008.

Enviado: Outubro, 2019.

Aprovado: Outubro, 2019. 